\title{
Barrier and Self-Energy Effects in Manganite Tunneling Characteristics
}

\author{
V. SVISTUNOV* \\ National Technical University, Frunze 21, Kharkov 61002, Ukraine \\ Y. Revenko, M. Belogolovskit, A. Gerasimenko, \\ Y. PAShKeVICH \\ A. Galkin Donetsk Physical and Technical Institute \\ National Academy of Sciences of Ukraine, 83114 Donetsk, Ukraine \\ H. Szymczak, P. Przyslupski and I. Komissarov \\ Institute of Physics, Polish Academy of Sciences \\ al. Lotników 32/46, 02-668 Warszawa, Poland

\begin{abstract}
Tunneling conductance spectra of point-contact junctions formed by a sharp Ag tip and two optimally doped manganites $\mathrm{La}_{0.67} \mathrm{Sr}_{0.33} \mathrm{MnO}_{3}$ and $\mathrm{Nd}_{0.67} \mathrm{Sr}_{0.33} \mathrm{MnO}_{3}$ are measured and analyzed. It was found that the analytical dependence of the differential conductance on voltage strongly depends on the bias sign and dramatically changes after voltage treatments. The data obtained are explained in terms of the Glasman-Matveev model for tunneling across two localized states settled in the barrier near the manganite bulk. Effective functions of electron-boson interaction extracted from self-energy contributions to the experimental characteristics contain signs of phonon and magnon excitations, higher-energy satellites as a result of possible orbital ordering and a hallmark of localized states in the near-barrier region in the form of strong not-well reproduced peaks.
\end{abstract}

PACS numbers: 75.47.Gk, 75.47.Lx

\section{Introduction}

Mixed-valence perovskite manganites of the form $\mathrm{R}_{1-x} \mathrm{~A}_{x} \mathrm{MnO}_{3}$ with $\mathrm{R}$, a rare-earth ion, and $\mathrm{A}$, a divalent ion, belong to a class of strongly correlated elec-

* corresponding author; e-mail: svistunov@kpi.kharkov.ua 
tron system near a metal-insulator transition. In the compounds, the competition between different ordering tendencies leads to complexity: their properties change dramatically upon the application of relatively small perturbations [1]. In particular, it concerns electric-field effects that manifest itself in a strong dependence of differential conductance on applied current bias and abrupt current-enforced resistivity jumps. What we want to emphasize here is that the resistance-versus-voltage curves often take a non-analytical form that was successfully described by a Glasman-Matveev (GM) model of an indirect tunneling [2] in a variety of lanthanum-based manganite systems: bulk ceramic samples [3], thin polycrystalline films [4], and low-doped single crystals [5]. In all cases the unusual conductance behavior was attributed to the appearance of intrinsic tunnel junctions with localized states inside an insulating barrier. On the non-analytical background the phenomena of current-induced instabilities and conductance jumps [6,7] as well as resistivity switching [5] have been observed. In our previous publication [8] we reported similar effects for junctions formed by a silver counter-electrode with manganite ceramics and related them to inhomogeneities in the barrier and near-barrier regions of the material under study. Below we shall continue the analysis of the tunneling characteristics of optimally doped manganites $(\mathrm{R}=\mathrm{La}$ and $\mathrm{Nd}, \mathrm{A}=\mathrm{Sr}$ ) in a broad range of bias voltages. Because, in contrast to the cited works, we are dealing with an asymmetrical structure, we find some significant differences, in particular, in the influence of different voltage polarities that provide us with an opportunity to know about the location of localized states. We also present the data for an effective function of electron-boson interaction in the manganites measured that confirm the presence of the states near the manganite surface.

\section{Experimental}

Thin films of $\mathrm{La}_{0.67} \mathrm{Sr}_{0.33} \mathrm{MnO}_{3}$ and $\mathrm{Nd}_{0.67} \mathrm{Sr}_{0.33} \mathrm{MnO}_{3}$ were fabricated on (100)-oriented $\mathrm{LaAlO}_{3}$ substrates (heated at $760-770^{\circ} \mathrm{C}$ in 3 mbar oxygen pressure) by a high-pressure dc sputtering method. Their electrical, magnetic, and microwave properties were characterized in the paper [9]. The system studied experimentally was a point-contact junction formed with a sharp Ag tip pressed against the sample with a force that could be regulated by an adjusting mechanism. The derivatives of the current $(I)$-versus-voltage $(V)$ curves were measured by a standard modulation technique in the fixed-voltage regime. The second derivative $\mathrm{d}^{2} I(V) / \mathrm{d} V^{2}$, which was used for the extraction of the effective spectral function of electron-boson interaction according to the method described in detail in Ref. [10], was obtained by numerical differentiation of the experimentally measured and numerically smoothed differential conductance $\mathrm{d} I(V) / \mathrm{d} V$. Junction resistance jumps were initiated by applying voltage biases greater than $1 \mathrm{~V}$ as it was explained in our previous work [8]. 


\section{Results and discussion}

Figure 1 illustrates the evolution of $\mathrm{d} I(V) / \mathrm{d} V$ characteristics for a junction with the neodymium manganite film after two successive positive-voltage treatments caused a decrease in the junction resistance in comparison with the differential conductance of $\mathrm{La}_{0.67} \mathrm{Sr}_{0.33} \mathrm{MnO}_{3}$. Two main features should be stressed: (i) there is no symmetry with respect to the bias voltage; (ii) the character of the conductance spectrum radically changes after several applications of high voltages; (iii) there is an apparent difference between tunneling characteristics for lanthanum- and neodymium-based manganites. The last finding reminds the correlation between the slope of tunneling spectra of copper oxide-based junctions and the normal-state properties of high-temperature superconducting perovskites [11]. Below we shall dwell on the former two observations. Let us try to describe $\mathrm{d} I(V) / \mathrm{d} V$ curves from Fig. 1 separately for both polarities by a simple formula

$$
\sigma(V)=\sigma_{0}+\alpha V^{\delta}
$$

with $\sigma_{0}=\sigma(V=0)$, a dimensionless voltage $V$, and $\delta$ varying from $\delta=4 / 3$ (tunneling through 2 localized states [2]) to $\delta=2$ (conventional direct tunneling across a rectangular potential barrier). The last statement means that we assume the presence of a symmetric barrier (an asymmetry reveals itself in an offset of a conductance minimum from $V=0$ that in our case was negligibly small) with some states inside it. The latter ones may be localized at any part of the barrier and, as will be proved below, it is a source of a strong asymmetry of our curves. As we believe, the voltage treatment changes the number of the centers inside the

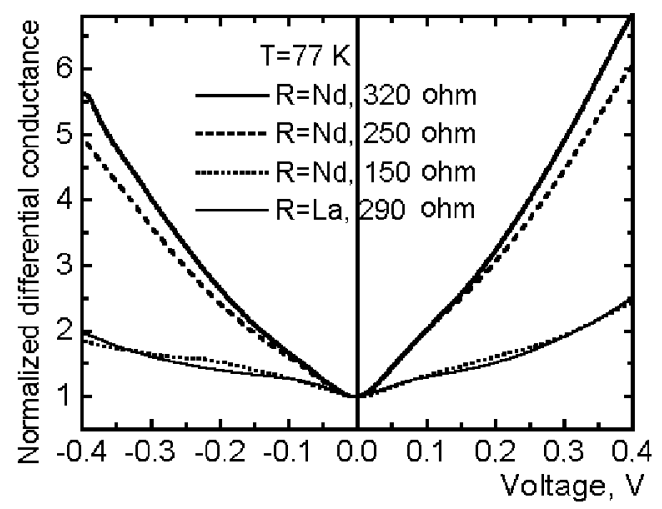

Fig. 1. Normalized tunneling conductance spectra for $\mathrm{Ag} / \mathrm{Nd}_{0.67} \mathrm{Sr}_{0.33} \mathrm{MnO}_{3}$ (thick solid line) and $\mathrm{Ag} / \mathrm{La} 0.67 \mathrm{Sr}_{0.33} \mathrm{MnO}_{3}$ (thin solid line) contacts measured at $T=77 \mathrm{~K}$. Dashed and dotted curves are measured after the voltage treatment. Let us note that the initial resistances of both junctions are almost the same. Positive biases here and below correspond to a positive potential placed on the silver counter-electrode. 
forbidden space and their location that manifests itself in radical modifications of $\sigma(V)$ observable in Fig. 1.

Figures 2 and 3 show the dependence of $\ln \left[\left(\sigma(V)-\sigma_{0}\right) / \alpha\right]$ upon $\ln V$ for $\mathrm{Nd}_{0.67} \mathrm{Sr}_{0.33} \mathrm{MnO}_{3}$ and $\mathrm{La}_{0.67} \mathrm{Sr}_{0.33} \mathrm{MnO}_{3}$, respectively. It is evident that for nitrogen temperatures, in general, the behavior of two branches of the same conductance spectrum greatly differs from each other. If that for positive biases more resembles the GM model prediction with $\delta=4 / 3$ [2], the corresponding characteristic calculated for negative voltages more follows an analytic quadratic $(\delta=2)$ voltage dependence than it follows from the standard tunneling theory. But after two successive positive-voltage applications to a neodymium-based manganite junction both curves are drawing together. The same tendency is observed when the temperature is lowered to several K (Fig. 3). To explain the observations, we note that in our case the barrier structure is strongly asymmetric (in contrast with a symmetric GM model [2]) and the localization centers, if they are, should be settled near the manganite bulk. When electrons are injected from a counter-electrode into the bulk (it is the case of negative voltages), the main contribution comes from tunneling processes. In the opposite situation, the conductance spectrum is formed greatly by transferring across the localized states, as it can be seen in Fig. 2. With increasing temperature more and more the states are involved in the transport that should manifest itself in an enhancement of the parameter $\delta$. Because of

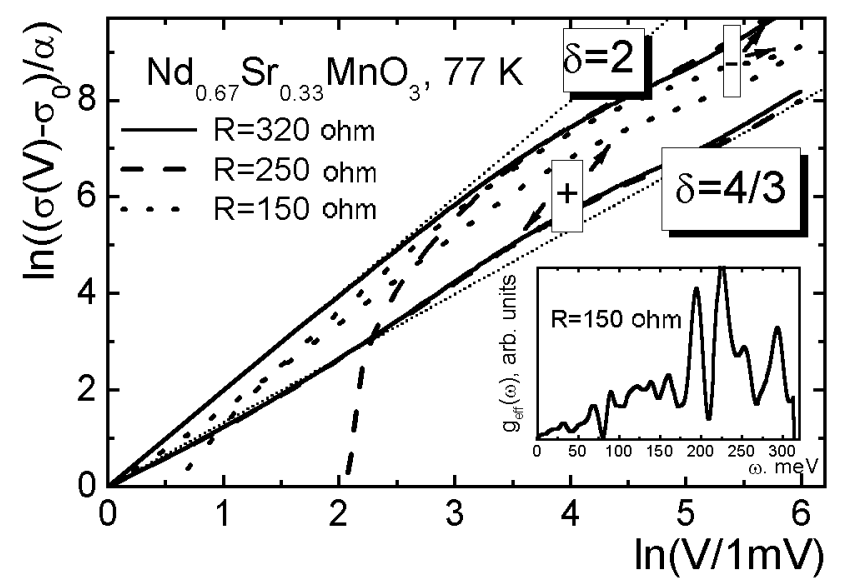

Fig. 2. Barrier characteristics of $\mathrm{Ag} / \mathrm{Nd}_{0.67} \mathrm{Sr}_{0.33} \mathrm{MnO}_{3}$ junctions at $T=77 \mathrm{~K}$ for both polarities indicated by signs "+" and "-". Initial spectra and those after the positive-voltage treatment are shown by solid, dashed, and dotted curves, respectively. Thin dotted straight lines represent a conventional voltage dependence of the tunnel conductance $(\delta=2)$ and that predicted by a GM model [2] for an indirect tunneling $(\delta=4 / 3)$. The inset shows an effective spectral function of electron-boson interaction reconstructed from the dotted-curve characteristics in the main panel. 


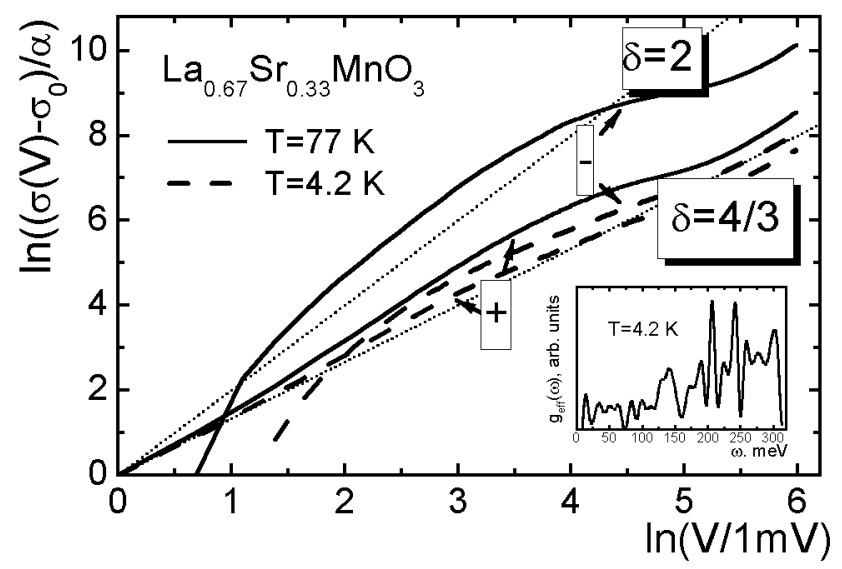

Fig. 3. Barrier characteristics of $\mathrm{Ag} / \mathrm{La}_{0.67} \mathrm{Sr}_{0.33} \mathrm{MnO}_{3}$ junctions at $77 \mathrm{~K}$ (solid curves) and $4.2 \mathrm{~K}$ (dashed curves) for both polarities indicated by signs "+" and "-". Thin dotted straight lines represent a conventional voltage dependence of the tunnel conductance $(\delta=2)$ and that predicted by a GM model [2] for an indirect tunneling $(\delta=4 / 3)$. The inset shows an effective spectral function of electron-boson interaction reconstructed from the data for $T=4.2 \mathrm{~K}$.

this, we expect that for lower temperatures the $\delta=4 / 3$ effect will become more pronounced that is confirmed by measurements shown in Fig. 3.

Earlier, we proposed a method of extracting the effective function of the electron-boson interaction $g_{\mathrm{eff}}(\omega)$ from self-energy effects in normal tunneling data that was described and applied to manganites in our previous paper [12]. The approach uses an odd part of the differential conductance and, to avoid undesirable contributions, it is necessary to deal with more symmetrical experimental data. That is why we have used for this purpose the spectrum measured for an $\mathrm{Ag} / \mathrm{Nd}_{0.67} \mathrm{Sr}_{0.33} \mathrm{MnO}_{3}$ junction with $R=150 \Omega$ and that for the $\mathrm{Ag} / \mathrm{La}_{0.67} \mathrm{Sr}_{0.33} \mathrm{MnO}_{3}$ device at $4.2 \mathrm{~K}$. The obtained $g_{\mathrm{eff}}(\omega)$ are shown in the insets in Figs. 2 and 3. As it was argued in Ref. [12], below $80 \mathrm{meV}$ we believe to observe the bosonic excitation spectrum of a manganite including phonons and magnons. A structure in the voltage region between 80 and $170 \mathrm{meV}$ could be explained either as harmonics of lattice vibration energies or as mixed modes of both orbital and phonon origin [12]. Badly reproducible peaks above $180 \mathrm{meV}$ may be caused by the metastable states studied in Refs. [5] and [7].

\section{Conclusion}

In summary, we have found strongly different behavior of two branches of manganite tunneling conductance spectra and explained it in terms of the Glasman-Matveev model [2] for tunneling across two localized states settled near 
the material bulk. Effective spectral functions of electron-boson interaction have been extracted from the experimental data for lanthanum- and neodymium-based manganite films. A structure between 80 and $170 \mathrm{meV}$ may be attributed to orbital ordering in the upper layers of the compounds studied whereas high-energy features which are not well reproduced are believed to reflect the presence of localized states in the near-barrier region.

\section{Acknowledgments}

This work supported by the State Committee for Scientific Research (Poland) under grants PBZ-KBN-013/T08/19 and 5P03B 06220.

\section{References}

[1] Colossal Magnetoresistance Oxides, Ed. Y. Tokura, Gordon and Breach, New York 2000; E. Dagotto, Nanoscale Phase Separation and Colossal Magnetoresistance, Springer-Verlag, Berlin 2002.

[2] L.I. Glazman, K.A. Matveev, Zh. Eksp. Teor. Fiz. 94, 332 (1988) [Sov. Phys.-JETP 67, 1276 (1988)].

[3] K.B. Chashka, B. Fisher, J. Genossar, L. Patlagan, G.M. Reisner, E. Shimshoni, Phys. Rev. B 63, 064403 (2001).

[4] M. Ziese, Phys. Rev. B 60, R738 (1999).

[5] Y. Yuzhelevski, V. Markovich, V. Dikovsky, E. Rosenberg, G. Gorodetsky, G. Jung, D.A. Shulyatev, Ya.M. Mukovskii, Phys. Rev. B 64, 224428 (2001).

[6] A. Asamitsu, Y. Tomioka, H. Kuwahara, Y. Tokura, Nature 388, 50 (1997); J. Stankiewicz, J. Sese, J. Garcia, J. Blasco, C. Rillo, Phys. Rev. B 61, 11236 (2000); S. Srivastava, N.K. Pandey, P. Padhan, R.C. Budhani, Phys. Rev. B 62, $13868(2000)$.

[7] V. Dikovsky, Y. Yuzhelevski, V. Markovich, G. Gorodetsky, G. Jung, D.A. Shulyatev, Ya.M. Mukovskii, Phys. Rev. B 65, 144439 (2002).

[8] M.A. Belogolovskii, Yu.F. Revenko, A.Yu. Gerasimenko, V.M. Svistunov, E. Hatta, G. Plitnik, V.E. Shaternik, E.M. Rudenko, Fiz. Nizk. Temp. 28, 553 (2002) [Low Temp. Phys. 28, 391 (2002)].

[9] J. Wosik, L.-M. Xie, M. Strikovski, P. Przyslupski, M. Kamel, V.V. Srinivasu, S.A. Long, J.Appl. Phys. 91, 5384 (2002).

[10] V.M. Svistunov, M.A. Belogolovskii, O.I. Chernyak, A.I. Khachaturov, A.P. Kvachev, Zh. Eksp. Teor. Fiz. 84, 1781 (1983) [Sov. Phys. JETP 57, 1038 (1983)].

[11] A.M. Cucolo, R. Di Leo, A. Nigro, P. Romano, F. Bobba, Phys. Rev. B 54, R9686 (1996).

[12] V.M. Svistunov, Yu.F. Revenko, M.A. Belogolovskii, A.Yu. Gerasimenko, A.I. Khachaturov, Yu.G. Pashkevich, E. Hatta, J. Phys. Soc. Jpn. 72, 2124 (2003). 